\title{
Increased angiopoietin2 expression is associated with endothelial apoptosis and blood-brain barrier breakdown
}

\author{
Sukriti Nag ${ }^{1}$, Tripti Papneja ${ }^{1}$, Roopa Venugopalan ${ }^{1}$ and Duncan J Stewart ${ }^{2}$ \\ ${ }^{1}$ Toronto Western Research Institute and Department of Pathology, University Health Network, Toronto, \\ Canada and ${ }^{2}$ Departments of Laboratory Medicine and Pathobiology and Medicine, Terrence Donnelly Heart \\ Center, St Michael's Hospital (DJS), University of Toronto, Toronto, Canada
}

\begin{abstract}
Normal intracerebral and pial vessels show constitutive expression of angiopoietin (Ang) 1 in endothelium while weak Ang2 immunoreactivity is present in occasional vessels. In the early phase postinjury, blood-brain barrier (BBB) breakdown at the lesion site is associated with decreased endothelial Ang1 and increased Ang2 expression, raising the possibility that Ang2 may have a role in early BBB breakdown. In order to determine whether Ang2 can cause BBB breakdown, the effect of recombinant Ang2 on cerebrovascular permeability to horseradish peroxidase (HRP) was studied in normal rat cortex. As hypothesized, Ang2 produced significant BBB breakdown to HRP as compared with vehicle-injected control rats. Since Ang2 is reported to have proapoptotic activity, the possibility that Ang2 may be associated with endothelial apoptosis was investigated in the rat cortical cold injury model over a period of $6 \mathrm{~h}$ to 6 days postinjury. Perilesion and pial vessels showed evidence of endothelial apoptosis as demonstrated by active Caspase-3 localization and TUNEL staining. Dual labeling for Ang proteins and active Caspase-3 demonstrated endothelial colocalization of Ang2 with active caspase-3. These data suggest that following injury, Ang2 may play a role in BBB breakdown of perilesional vessels, and it may also be a factor in endothelial cell apoptosis that occurs at days 1 and 2 following the injury. Laboratory Investigation (2005) 85, 1189-1198. doi:10.1038/labinvest.3700325; published online 1 August 2005
\end{abstract}

Keywords: active Caspase-3; angiogenesis; angiopoietins; apoptosis; Bax; blood-brain barrier; horseradish peroxidase

Angiopoietins (Ang) belong to a novel family of endothelial growth factors that function as ligands for the endothelial cell-specific receptor tyrosine kinase, Tie2. This family comprises three structurally related proteins, Ang1, Ang2 and Ang3/4 $4^{1-3}$ but best characterized are Ang1 and Ang2., ${ }^{4,5}$ Ang1 induces autophosphorylation of Tie2 whereas Ang2 competitively inhibits this effect, suggesting that it may be a naturally occurring inhibitor of Ang1/Tie2 activity. ${ }^{2}$ Ang1 and Ang2 are reported to have many reciprocal effects. Studies of cultured non-neural endothelial cells show that Ang1 has an antiapoptotic effect on endothelium, ${ }^{6-8}$ while Ang2 is reported to promote apoptosis. ${ }^{9,10}$ Ang1 stabilizes vessels by promoting the association of the vascular endothelium with perivascular

Correspondence: Dr S Nag, MD, PhD, McL-405, Neuropathology, Toronto Western Hospital, 399 Bathurst Street, Toronto, Ontario M5T 2S8, Canada.

E-mail: nag@uhnres.utoronto.ca

Received 27 May 2005; revised 23 June 2005; accepted 27 June 2005; published online 1 August 2005 cells $^{11,12}$ while Ang2 destabilizes vessels and in the presence of vascular endothelial growth factor-A (VEGF-A) leads to robust angiogenesis while in the absence of VEGF-A or basic fibroblast growth factor, vessel regression results. ${ }^{13,14}$ In addition, Ang1 has a strong leakage-resistant effect on endothelium of non-neural vessels ${ }^{15,16}$ and can overcome the permeability brought about by VEGF-A. ${ }^{16}$ Whether Ang2 can increase vascular permeability is not known.

Normal intracerebral and pial vessels show constitutive expression of Ang1 protein in endothelium while weak Ang2 immunoreactivity is present in occasional vessels. ${ }^{17}$ Our previous study of the rat cortical cold injury model demonstrated decreased or loss of endothelial Ang1 protein localization in lesion vessels showing blood-brain barrier (BBB) breakdown ${ }^{17}$ in the early phase postinjury. At this time period there was a concomitant marked increase in Ang2 expression at the gene and protein level raising the possibility that Ang2 may have a role in early BBB breakdown. 
In order to determine whether Ang2 can cause BBB breakdown, the effect of intracortical injections of recombinant Ang2 on cerebrovascular permeability to horseradish peroxidase (HRP) was studied in normal rat cortex. Recombinant VEGF-A, a known inducer of BBB breakdown, ${ }^{18-20}$ was also injected intracortically and served as a positive control in this study. The areas of HRP extravasation produced by both these agents and the density of the areas of extravasation were quantitated and compared with those of control rats.

Since Ang2 is reported to have proapoptotic activity, the possibility that Ang2 is associated with endothelial apoptosis was also investigated in the rat cortical cold injury model over a period of $6 \mathrm{~h}$ to 6 days postinjury. The time course of endothelial apoptosis was established by the terminal deoxynucleotidyl-transferase (TdT)-mediated dUTP-biotin nick end-labeling (TUNEL) method and immunohistochemical localization of the proapoptotic molecules Bax and active Caspase- 3 proteins. Further, in order to relate endothelial apoptosis with Ang protein expression in lesion vessels, dual labeling was done at days 1 and 2 postinjury using the TUNEL method followed by immunohistochemical localization of Ang1 or Ang2. In addition, dual labeling for Ang1 or Ang2 and active Caspase-3 proteins was also performed.

\section{Materials and methods}

Protocols involving the use of rats were in accordance with the guidelines set by the Canadian Council on Animal Care and were further approved by the local animal care committee.

\section{Intracortical Injection Model}

Male Wistar-furth rats (180-200 g) were anesthetized by an i.p. injection of Sodium Amytal $80 \mathrm{mg} / \mathrm{kg}$. A craniotomy was performed in the left parietal bone using a dental drill having a $1.2 \mathrm{~mm}$ bit. A Hamilton syringe mounted on a micromanipulator was used to inject $400 \mathrm{ng}$ of recombinant mouse VEGF-A (R\&D Systems, Minneapolis, MN, USA) and 100 and $400 \mathrm{ng}$ of Ang2 (R\&D Systems) in $15 \mu \mathrm{l}$ of sterile phosphate-buffered saline containing $0.1 \%$ bovine serum albumin, in separate groups of 10 rats per group. Control rats were injected with the same vehicle as used for the test injections. The injection needle was lowered into the cortex for a distance of $2 \mathrm{~mm}$ and the required substance was injected slowly over a period of $2 \mathrm{~min}$. The needle was left in place for $1 \mathrm{~min}$ and slowly withdrawn over $2 \mathrm{~min}$ to prevent reflux of the injected solutions. Test and control rats were killed $1 \mathrm{~h}$ after the injection. At 10 min prior to killing, HRP Type II (Sigma Chemical Co., St Louis, MO, USA, $15 \mathrm{mg} / 200 \mathrm{~g}$ ) was injected i.v. via a polyethylene cannula in the femoral artery. Rats were perfused with a mixture of $2 \%$ parafor- maldehyde and $0.5 \%$ glutaraldehyde in $0.1 \mathrm{M}$ phosphate buffer $(\mathrm{pH}$ 7.3) at a pressure of $110 \mathrm{mmHg}$, by a cannula in the ascending aorta.

Brains of rats were removed and sectioned at $50 \mu \mathrm{m}$ intervals using a Vibratome. Brain slices were reacted for the demonstration of HRP reaction product as described previously. ${ }^{21}$

Brain slices were mounted in glycerine and examined by light microscopy.

\section{Cortical Cold-Injury Model}

Cold injury was produced in male Wistar rats (180$200 \mathrm{~g}$ ) as described previously. ${ }^{22}$ Rats were anesthetized by methoxyflurane inhalation. After a skin incision, a craniotomy was performed in the left parietal bone using a dental drill with a bit measuring $2.3 \mathrm{~mm}$ in diameter. A cold probe consisting of the tip of a copper wire, contained in a $20 \mathrm{ml}$ syringe containing liquid nitrogen, was placed over the brain at the craniotomy site for $45 \mathrm{~s}$. The skin incision was approximated with metal clips and rats were allowed to recover. Rats were killed in groups of five at $6 \mathrm{~h}$ and 1 day intervals over a period of 1-6 days. Immunohistochemistry and in situ detection of DNA fragmentation by the TUNEL method were done as follows:

\section{Immunohistochemistry}

At the required time periods stated above, coldinjured and control rats were anesthetized with Metofane and perfused with $3 \%$ paraformaldehyde in $0.1 \mathrm{M}$ phosphate buffer ( $\mathrm{pH}$ 7.3). A coronal slab of brain containing the cold injury site was processed for paraffin sectioning using standard techniques. Sections $(6 \mu \mathrm{m})$ were stained with hematoxylin and eosin for histological analysis and adjacent sections were used for immunohistochemistry.

The indirect streptavidin-biotin peroxidase method was used and paraffin sections were pretreated with $0.5 \%$ pepsin in $0.01 \mathrm{M} \mathrm{HCl}$ for $30 \mathrm{~min}$ at $37^{\circ} \mathrm{C}$ before overnight incubation in primary antibody at $4^{\circ} \mathrm{C}$. The source of polyclonal antibodies and the dilutions used were: Ang1 and Ang2 (Santa Cruz Biotechnology, CA, USA) 1:50; Fibronectin (Gibco BRL) 1:400; active Caspase 3 (R\&D Systems) 1:1200 and Bax (Santa Cruz Biotechnology) 1:1500.

Dual-labeling immunohistochemistry was also performed at days 1 and 2 postinjury for (1) Ang2 and fibronectin, (2) Ang1 and active Caspase-3, and (3) Ang2 and active Caspase-3. Details of the duallabeling method have been described previously. ${ }^{23}$ Stretpavidin-Alexa Fluor ${ }^{\mathrm{TM}} 488$ (Molecular Probes Inc., Eugene, OR, USA) and goat antirabbit-Cy ${ }^{\mathrm{TM}} 3$ (Jackson Immunoresearch Laboratories Inc., Westgrove, PA, USA) were used as link antibodies and emitted a green and red fluorescence, respectively. Sections were analyzed using a Nikon Optiphot microscope and a MRC 600 confocal laser-scanning 
microscope (Bio-Rad laboratories Canada Ltd., Mississauga, Canada). Images were merged using Adobe Photoshop 7.0 software.

Negative controls included omission of primary antibody, neutralization of the primary antibody with blocking peptides, and using nonimmune serum. Positive controls used were the lung for Ang1, the prostate for Ang2, and the ovary for both Ang1 and Ang2.

\section{In Situ Detection of DNA Fragmentation}

TUNEL-positive cells were detected as described previously ${ }^{24}$ with minor modifications. Following deparaffinizaton, sections were washed with distilled water and placed successively in the following solutions: (1) $50 \mathrm{mM}$ Tris-HCl buffer ( $\mathrm{pH}$ 7.0) containing $5 \mathrm{mM} \mathrm{CaCl}_{2}$ at room temperature (RT) for $5 \mathrm{~min}$, (2) Proteinase K $(25 \mu \mathrm{g} / \mathrm{mL}$, Life Technologies) in the same buffer as (1) for $15 \mathrm{~min}$ at RT, (3) TdT buffer ( $\mathrm{pH}$ 6.6) containing $1 \mathrm{M} \mathrm{Na}$ cacodylate, $1 \mathrm{M}$ Tris-HCl, $1.6 \mathrm{mg} / \mathrm{ml}$ bovine serum albumin, and $1 \mathrm{mM} \mathrm{CoCl}$, for $15 \mathrm{~min}$, (4) TdT buffer containing TdT $0.05 \mathrm{U} / \mu \mathrm{l}$ buffer and $1 \mathrm{mM}$ biotin-16-dUTP (Roche Diagnostics) for $90 \mathrm{~min}$ in a humid chamber at $37^{\circ} \mathrm{C}$, (5) TB buffer containing $300 \mathrm{mM} \mathrm{NaCl}$ and $30 \mathrm{mM}$ sodium citrate, (6) distilled water rinse, (7) $1 \%$ hydrogen peroxide for $5 \mathrm{~min}$, (8) distilled water, (9) PBS containing 1\% BSA for $10 \mathrm{~min}$ at RT, (10) distilled water, then PBS, (11) streptavidin peroxidase (1:650 dilution, Jackson Immunoresearch Laboratories) for $30 \mathrm{~min}$ at RT, (12) PBS rinse, (13) $0.06 \%$ diaminobenzidine in Tris-saline containing $0.02 \%$ hydrogen peroxide, and (14) $50 \%$ hematoxylin for $45 \mathrm{~s}$. Brain sections of the lesion and contralateral cortex incubated without the enzyme or the nucleotide were used as negative controls and sections of glioblastoma multiforme were used as positive controls.

\section{Quantitation and Statistical Analyses}

The following quantitation was done using a MCID Image Analyser (Imaging Research Inc., St Catherines, Ontario, Canada): (1) the areas of HRP extravasation at the injection site produced by VEGF-A, Ang2 and vehicle-injected control rats were measured in brains of 10 rats per group. The areas of the needle track and hemorrhage were also measured and excluded from the value of the total area. Three vibratome sections of the lesion site containing the needle track were used per rat and for each section the area was measured three times and the mean value was calculated. (2) Mean density of the areas of HRP extravasation in these groups was obtained by using a tool measuring $10 \times 10$ pixels to measure the density of 12 constant locations within the area of HRP extravasation. The section background value was subtracted from the density value obtained. (3) Free cells within the lesion showing intense TUNEL staining excluding endothelial cells lining vessels were quantified at $6 \mathrm{~h}$ and 1 day intervals from 1 to 6 days using a $\times 250$ final magnification. Cells were counted in a defined area of $0.356 \mathrm{~mm}^{2}$ per lesion in each rat. These values were used to calculate the number of TUNELpositive cells per $\mathrm{mm}^{2}$ of lesion area. Three sections were used for each animal and data from three animals were used for each time point. (4) The total number of vessels showing endothelial active Caspase-3 immunostaining and those showing TUNEL staining in the entire lesion were counted at $6 \mathrm{~h}, 1$ and 2 days in three rats per group at a $\times 400$ total magnification. Counts were performed in a blinded manner to exclude observer bias.

Means were compared using the Mann-Whitney test (SPSS Science Inc., Chicago, Il, USA). Differences were considered significant at a value of $P<0.05$.

\section{Results}

\section{Ang2-Induced BBB Breakdown}

Brains of vehicle-injected control rats showed minimal HRP extravasation around the needle track (Figure 1a) and the mean area of extravasation in the 10 control rats was $0.21 \pm 0.01 \mathrm{~mm}^{2}$. Following an intracortical injection of VEGF-A, the mean area of HRP extravasation around the needle track was $0.40 \pm 0.02 \mathrm{~mm}^{2}$, a value which is significantly greater $(P<0.001)$ than the area of extravasation in vehicle-injected control rats (Figures 1b, 2a). An intracortical injection of $100 \mathrm{ng}$ of Ang2 did not produce significant HRP extravasation as compared with vehicle-injected control rats (Figure 1c). However, $400 \mathrm{ng}$ of Ang2 produced significantly larger areas of HRP extravasation $(P<0.001)$ as compared with vehicle-injected control rats and the mean area for this group was $0.39 \pm 0.01 \mathrm{~mm}^{2}$ (Figures 1d, 2a). Quantitation of the density of the areas of HRP extravasation in response to $400 \mathrm{ng}$ of either VEGF-A or Ang2 showed that the density of these areas was significantly greater than the density of areas of HRP extravasation in vehicle-injected control rat brains (Figure 2b).

\section{Morphology of the Cold Lesion}

The morphological changes at the lesion site, the time course of BBB breakdown and angiogenesis are similar to our previous observations. ${ }^{18,23,25,26}$ Immediately after production of the lesion, a central area of coagulative necrosis extends from the pial surface to the fourth cortical layer. Blood vessels in this central region are necrotic. Neuronal alterations are evident by $6 \mathrm{~h}$.

There are two phases of active BBB breakdown postinjury. An initial phase involves arterioles and large venules at the margins of the lesion starting 

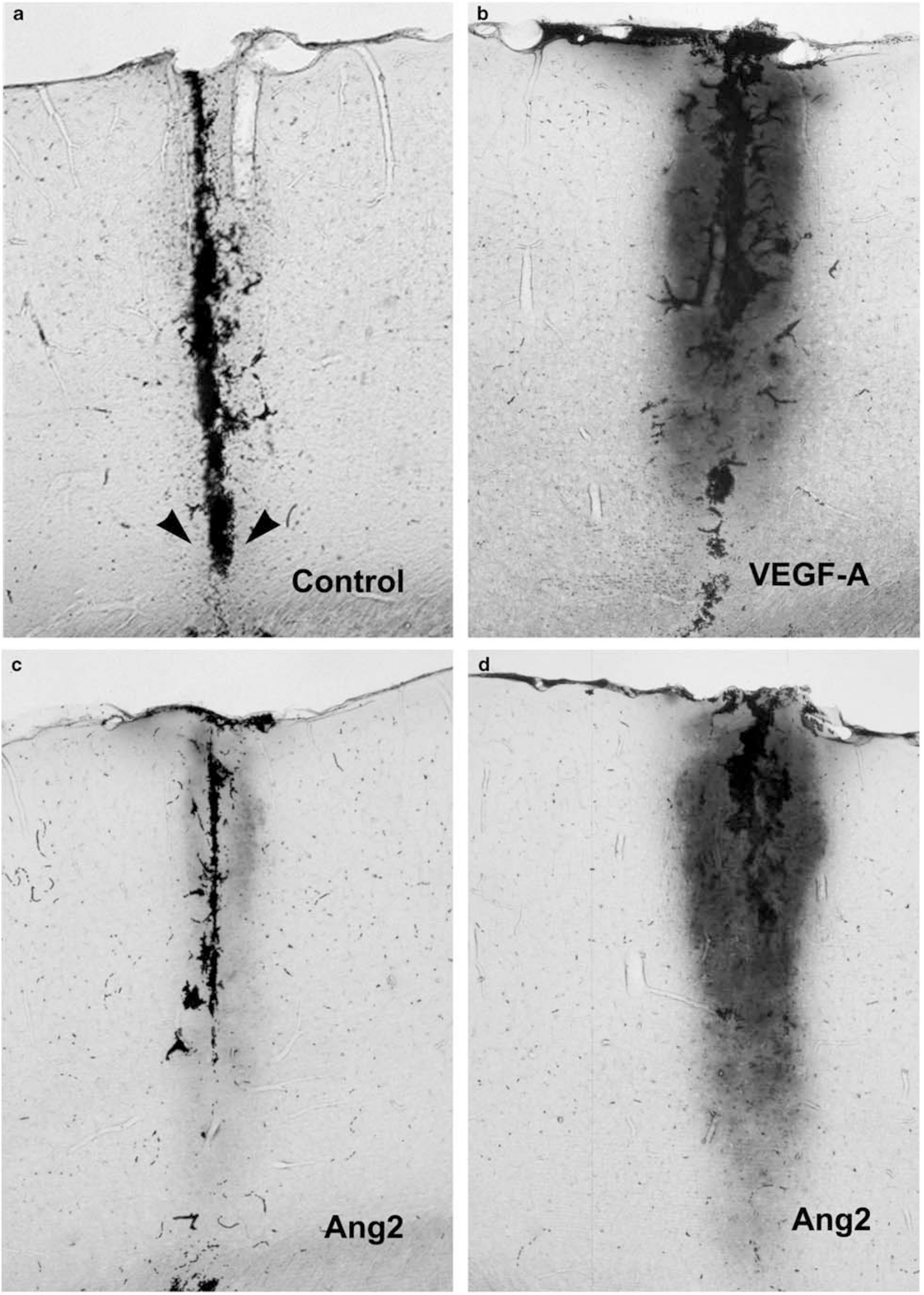

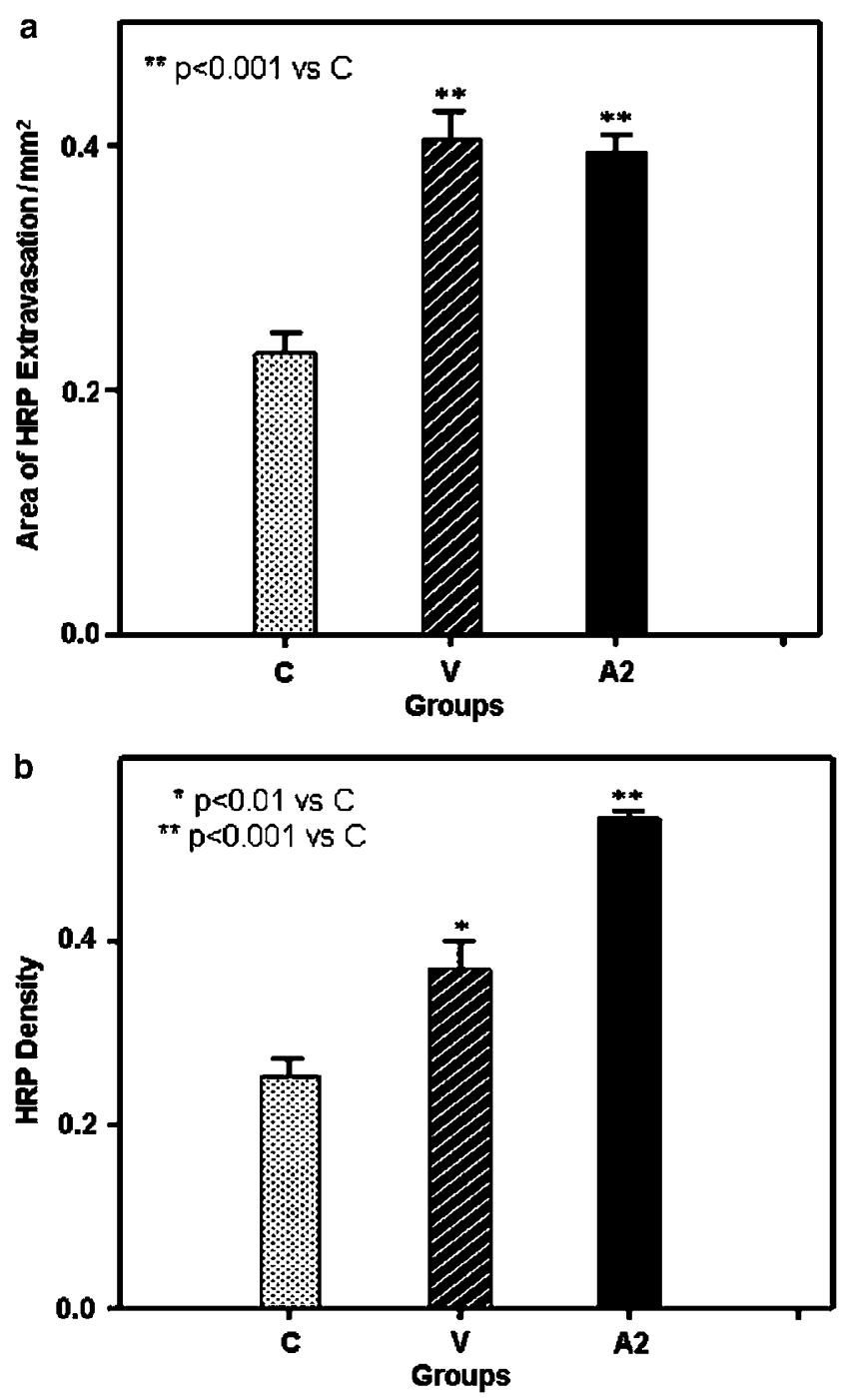

Figure 2 (a) Histogram showing the mean areas of HRP extravasation at $1 \mathrm{~h}$ in brains of vehicle-injected control rats $(\mathrm{C})$ and rats injected with $400 \mathrm{ng}$ of VEGF-A (V) or Ang2 (A2). Both VEGF-A and Ang2 produce significantly larger areas of HRP extravasation $(P<0.001)$ as compared with vehicle-injected control rats. (b) Histogram showing the density of HRP extravasation at $1 \mathrm{~h}$ in brains of vehicle-injected control rats and rats injected with $400 \mathrm{ng}$ of VEGF-A or Ang2. The density of the areas of HRP extravasation produced by VEGF-A and Ang2 are significantly greater, $(P<0.01$ and 0.001$)$ respectively, as compared with brains of vehicle-injected control rats. Values in (a) and (b) represent mean \pm s.e.m. of 10 rats per group.

from $1 \mathrm{~h}$ postinjury and results in massive protein extravasation into the white matter of the ipsilateral hemisphere and via the corpus callosum into the white matter of the contralateral hemisphere as well. The second phase of BBB breakdown occurs during the phase of angiogenesis and involves mainly neovessels resulting in edema localized to the lesion site.

The onset of angiogenesis is marked by cellular proliferation around pre-existing vessels at the margins of the lesion from day 2. These cells are immunoreactive with Factor VIII and Glut-1 antibodies confirming their endothelial nature and also show bromodeoxyuridine labeling. ${ }^{23}$ These cells will be referred to as proliferating endothelial cells in this paper. Some of these cells form vascular tubes and neovessels which are evident at day 4 . At day 6 the entire lesion is occupied by intrinsic vessels that survive the injury, neovessels, free endothelial cells which do not form neovessels and macrophages.

\section{Apoptosis at the Cold-Injury Site}

\section{Neuronal changes}

Cortical neurons in both hemispheres of control rats failed to show immunoreactivity for Bax or active Caspase-3 proteins or TUNEL staining.

Few neurons within the lesion showed active Caspase-3 and Bax immunoreactivity and TUNEL staining at $6 \mathrm{~h}$ while at day 1 all neurons within the lesion were strongly immunoreactive for Bax (Figure 3a) and stained by the TUNEL method (Figures 3c, d). TUNEL-stained neurons showed the morphological features of apoptosis with cell shrinkage and condensed nuclei. Quantitation demonstrated that the number of neurons showing TUNEL positivity was maximal at day 1 being $99 \pm 8 / \mathrm{mm}^{2}$ (Figure 4a). By day 2, fewer neurons were evident in the lesion and they still demonstrated Bax immunoreactivity as well as TUNEL staining. No neurons were observed in the lesion site after day 2 .

\section{Vascular changes}

The intracerebral vessels of both hemispheres of control rats failed to show immunoreactivity for Bax, active Caspase-3 proteins or TUNEL staining.

At day 1, maximal numbers of pial and lesion vessels showed Bax protein in both the endothelium and the smooth muscle cells (Figure 3b). Focal vascular segments showed Bax protein upto day 4. Endothelium of few pial and lesion vessels showed active Caspase- 3 at $6 \mathrm{~h}$. Maximal active Caspase- 3 immunoreactivity was observed in vessels at day 1 (Figure 3e, 4b), and the number of vessels showing immunoreactivity for active Caspase-3 decreased by day 2 (Figure $4 \mathrm{~b}$ ).

Figure 1 The intracortical injection model. (a) Cortex of a vehicle-injected control rat showing red blood cells in the needle track and minimal extravasation of HRP (arrowheads). At $1 \mathrm{~h}$, there is significant extravastion of HRP in response to $400 \mathrm{ng}$ of recombinant VEGF-A (b). At $1 \mathrm{~h}$, a mild increase in HRP extrvasation is present in response to $100 \mathrm{ng}$ of Ang2 (c) while a large area of HRP extravasation is present around the needle track in the cortex of a rat injected with $400 \mathrm{ng}$ of recombinant Ang2 (d). (a)-(d), $\times 50$. 


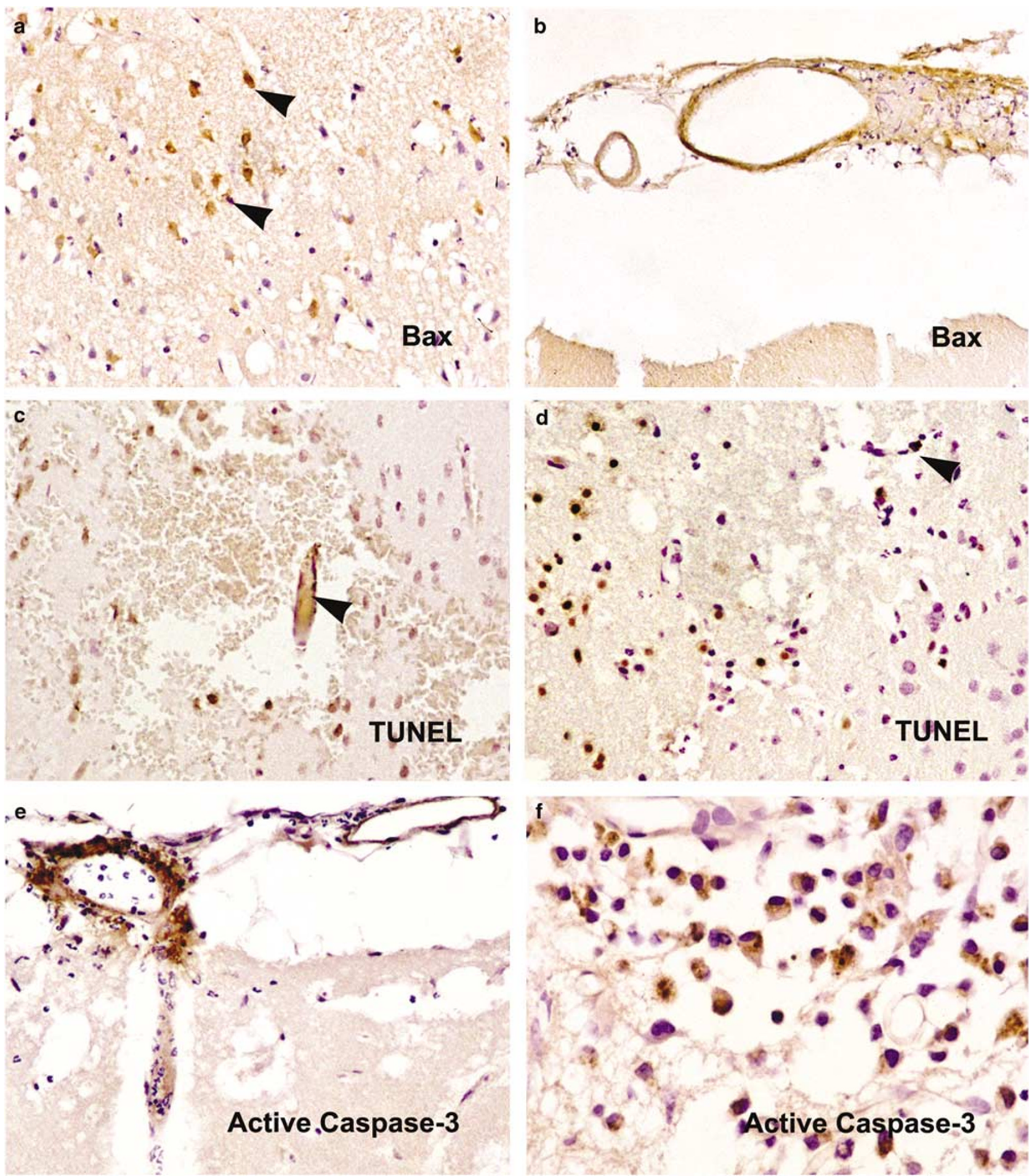

Figure 3 Bax protein is shown in neurons (arrowheads) at the lesion site (a) and in the wall of a pial vessel at day 1 (b). TUNEL staining in neurons at the left margins of the lesions at $6 \mathrm{~h}(\mathbf{c})$ and day $1(\mathbf{d})$ are shown. Also present is TUNEL staining in endothelial cells of a penetrating arteriole (arrowhead, c) and a microvessel (arrowhead, d). (e) A pial arteriole and adjacent penetrating arteriole shows mural active Caspase-3 immunostaining at day 1. (f) Macrophages from a day 3 lesion show cytoplasmic active Caspase-3. (a-e) $\times 300$; (f) $\times 650$.

Lesion vessels with TUNEL-positive endothelial cells were seen as early as $6 \mathrm{~h}$ (Figures 3c, d) after injury and their number progressively increased with peak values at day 2 after injury (Figure 4b).
As reported previously, ${ }^{17}$ dual labeling for Ang2 and fibronectin demonstrated endothelial Ang2 in pial and fewer perilesional vessels showing BBB breakdown to fibronectin (Figures 5a, b). The 

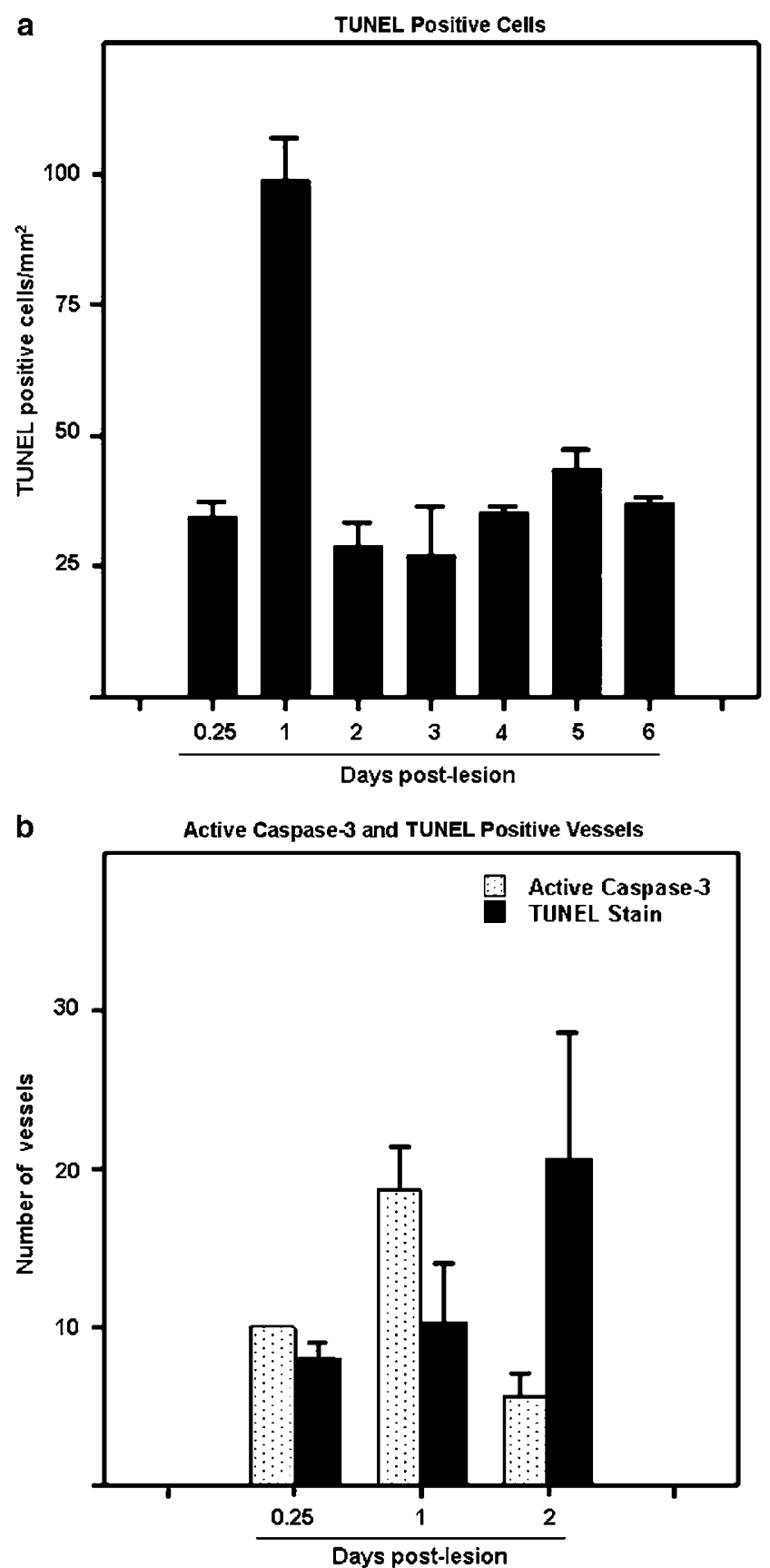

Figure 4 (a) Histogram showing the number of free TUNELpositive cells in the lesion per $\mathrm{mm}^{2}$ of lesion tissue at $0.25-6$ days excluding the TUNEL-positive endothelial cells lining vessels which are shown in (b). The number of TUNEL-positive cells at day 1 represents apoptosis of all neurons within the lesion. After day 2 the labeled cells are mainly endothelial and inflammatory cells. Maximal TUNEL labeling of these cells are noted at day 5 which is past the peak of vascular tube formation which occurs at day 4. (b) Histogram showing the mean number of active Caspase3 positive vessels and vessels with endothelial TUNEL staining at the lesion site at $0.25,1$ and 2 days. The values shown represent the mean value \pm s.e.m. of three rats per group. Maximal endothelial active Caspase-3 immunoreactivity is present at day 1 while the maximal endothelial TUNEL staining is present at day 2 .

TUNEL technique followed by immunohistochemical detection of Ang1 or Ang2 gave poor results due to cytoplasmic loss following treatment of sections with Proteinase-K. Therefore dual-labeling immunohistochemistry was performed for Ang1 or Ang2 and active Caspase-3. At day 1, a mean of $19 \pm 3$ pial or intracerebral lesion vessels showed immunoreactivity with active Caspase in both the endothelial and smooth muscle layer (Figure 4b). Occasional active Caspase- 3 positive vessels showed Ang-1 in focal segments of endothelium (Figure 5d) but most of these vessels showed no endothelial Ang1 while endothelial colocalization of active Caspase- 3 and Ang2 was noted in many vessels (Figures 5e, f).

\section{Angiogenesis}

The increased numbers of cells at the lesion site have previously been demonstrated to be both endothelial cells and macrophages. ${ }^{25,26}$ Both cell types were immunoreactive for active Caspase-3 at days 3 and 4 (Figure 3f). Endothelial cells and macrophages showing TUNEL staining increased from a mean of $27 \pm 9$ cells $/ \mathrm{mm}^{2}$ at day 3 to $44 \pm 4$ cells $/ \mathrm{mm}^{2}$ at day 5 and then decreased at day 6 to a mean of $37 \pm 1$ cells $/ \mathrm{mm}^{2}$ (Figure $4 \mathrm{a}$ ).

\section{Inflammatory cells}

Neutrophils infiltrating the lesion site at day 1 showed intense cytoplasmic immunoreactivity for active Caspase-3. Dual labeling showed colocalization of Ang-1 or Ang2 and active Caspase-3 in the infiltrating neutrophils (Figures 5d, f). Macrophages infiltrated the lesion site starting from day 1 and they showed cytoplasmic immunoreactivity for active Caspase-3 from days 3 up to 5 (Figure 3f).

\section{Discussion}

The novel findings in this study are that Ang2 can induce BBB breakdown and that following brain injury lesion vessels showing evidence of endothelial apoptosis as indicated by active Caspase-3 localization and TUNEL staining demonstrate endothelial Ang2 but not Ang1 suggesting that Ang2 may have a role in endothelial apoptosis.

\section{Ang2 and BBB Breakdown}

Our previous study of angiopoietin expression in the cold-injury model showed significant upregulation of Ang2 at $6 \mathrm{~h}$ when BBB breakdown of perilesion vessels is already present. ${ }^{17}$ In order to determine whether Ang2 can induce BBB breakdown, recombinant Ang2 was used to study BBB breakdown to HRP and recombinant VEGF-A, an agent known to produce hyperpermeability of cerebral vessels ${ }^{19}$ was used as a positive control to validate the model. This model was preferred to in vitro studies since one of the morphological determinants of the BBB namely, the interendothelial junctions of cultured cerebral endothelial cells are not as complex or 'tight' as those present in vivo. ${ }^{27}$ Qualitative and quantitative data 
demonstrate that Ang2 can induce BBB breakdown to HRP, producing larger areas of HRP extravasation as compared with vehicle-injected control rats. A variable amount of bleeding does occur in the
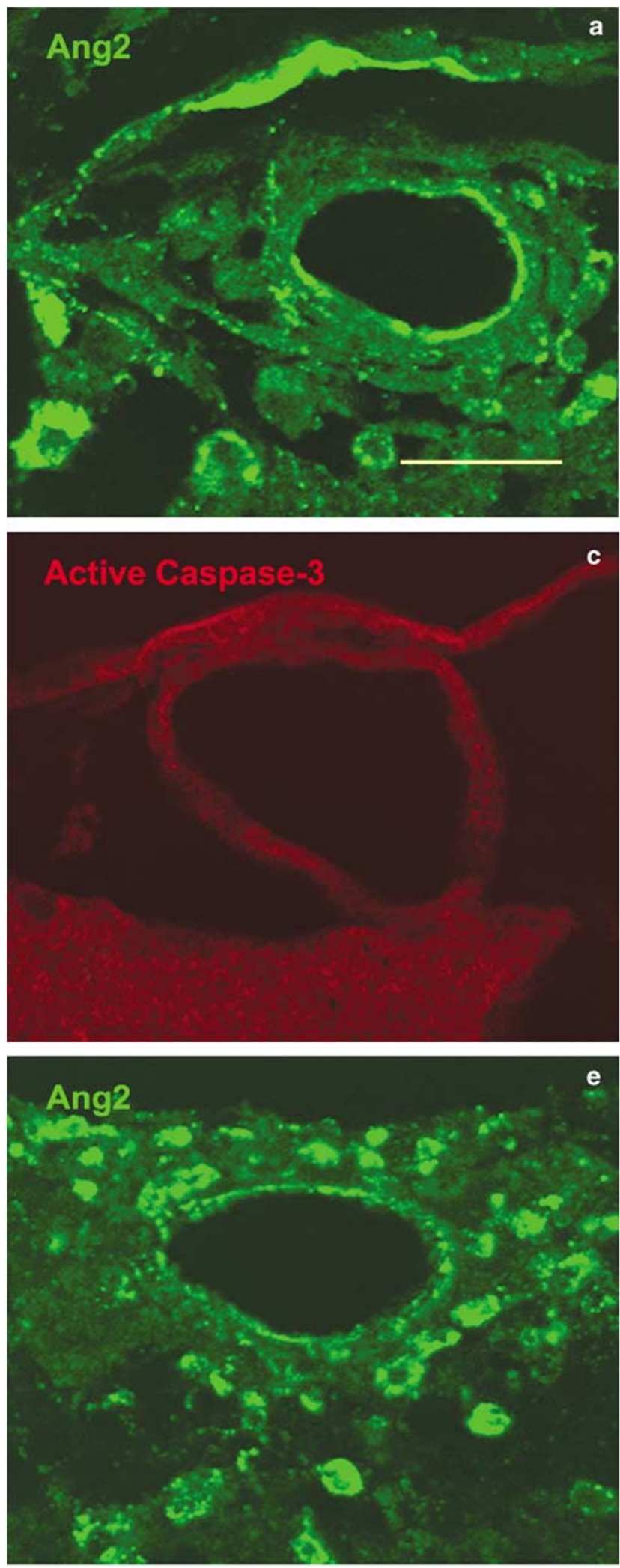

needle track therefore the area occupied by red blood cells was measured and excluded from the total area of HRP extravasation. The finding that Ang2 can induce BBB breakdown is novel and has
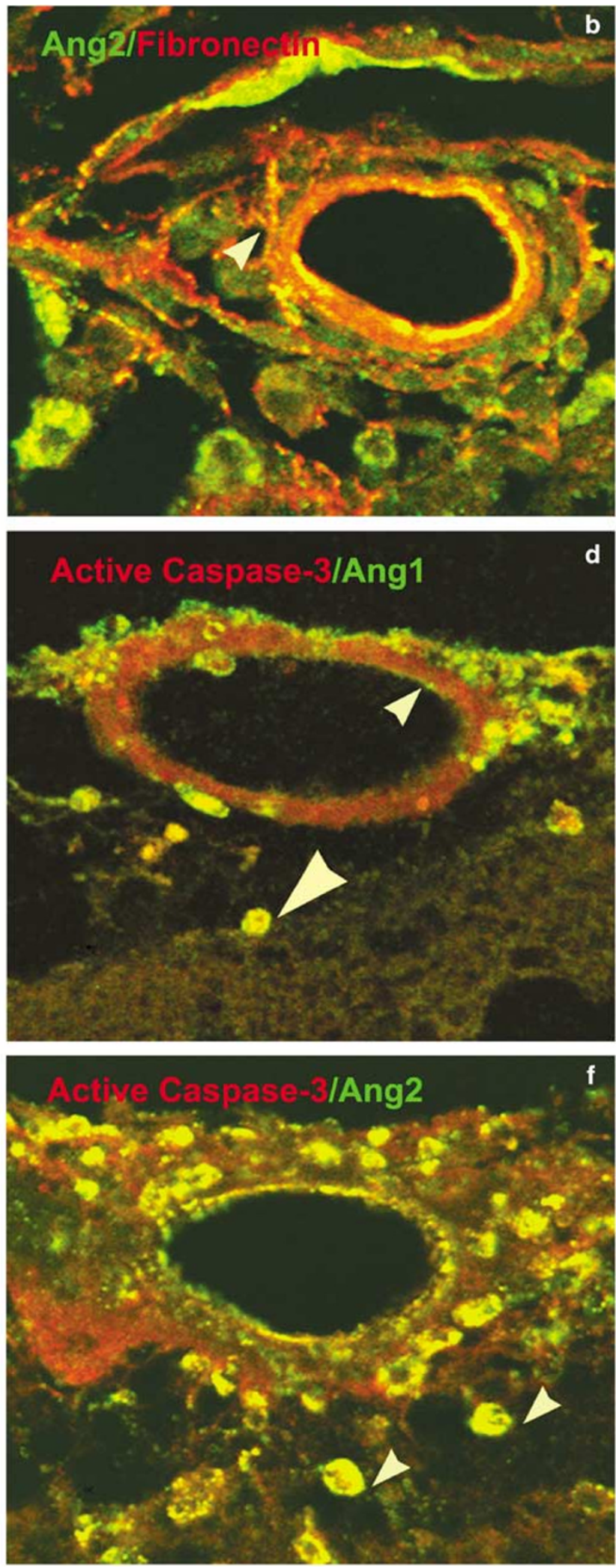
not been reported previously. Thus far, only the angiogenic and not the permeability effects of recombinant Ang2 has been studied in vitro and in non-neural endothelial cells. ${ }^{28}$

While the cerebral endothelial structural changes resulting in VEGF-mediated permeability are known, ${ }^{19}$ the endothelial structural changes induced by Ang2 leading to BBB breakdown remains to be determined.

\section{Apoptosis in the Cold-Injury Model}

Apoptosis is characterized by gene-directed processes in which new RNA and proteins are synthesized, which allow the selective elimination of cells. Several proapoptotic proteins were localized in this study because of the cautionary note that a positive TUNEL assay may not be a specific marker of apoptosis and could also indicate necrotic cell death. ${ }^{29}$

Other studies of cold-injury models that are not identical to the model used in the present study demonstrated neuronal apoptosis as early as $30 \mathrm{~min}$ after the injury by DNA electrophoresis, ${ }^{30,31}$ or the TUNEL method. ${ }^{30}$ In these studies apoptosis was maximal at day 1 similar to the observation in the present study which also demonstrated maximal TUNEL and Bax immunoreactivity at day 1 . There was no mention of endothelial cell apoptosis in these studies.

In this study, perilesion vessels showing endothelial active Caspase-3 were maximal at day 1 while those showing maximal TUNEL staining were maximal at day 2. These vessels also showed mural Bax protein supporting the presence of endothelial apoptosis. One other study has reported endothelial TUNEL staining in vessels in the infarct center at day 1 , following middle cerebral artery occlusion. ${ }^{32}$

Dual labeling showed that apoptotic vascular segments of lesion vessels which were active Caspase-3 positive also showed loss of Ang1 protein and endothelial colocalization of Ang2 at day 1 . Loss of Ang1, a potent antiapoptotic factor provides a mechanism for endothelial apoptosis. Increased concentrations of Ang2 are known to block Ang-1 activation of Tie2. ${ }^{2}$ Expression of Ang2 by endothelial cells represents an effective autocrine mechanism for delivering an antagonist to the receptor it was designed to block. The association of Ang2
mRNA-expressing endothelial cells with apoptotic endothelial cells has also been reported in the periinfarct area, following MCA occlusion. ${ }^{32}$ However, these authors could not investigate induction of Ang2 mRNA and apoptosis within the same sections due to technical reasons. Our previous studies demonstrated that VEGF-A mRNA is significantly increased only at day $3^{23}$ while Ang2 mRNA is significantly increased at $6 \mathrm{~h}$ following injury, ${ }^{17}$ therefore the early upregulation of Ang2 in the absence of VEGF-A could mediate endothelial apoptosis and vascular regression as has been suggested previously ${ }^{13,14}$ and thereby lead to $\mathrm{BBB}$ breakdown.

\section{Angiogenesis}

Programmed cell death plays an important role during development and tissue turnover by reinforcing appropriate cellular patterns or removing cells that are harmful or no longer needed. Proliferating endothelial cells demonstrate active Caspase- 3 at days 3 and 4 while TUNEL-positive endothelial cells are observed later at days 5 and 6 postinjury supporting the role of apoptosis in modulating angiogenesis in this model since endothelial cells that have not formed vascular tubes and immature neovessels consisting of only endothelial cells and no pericytes are known to regress in the later phases of angiogenesis. ${ }^{33}$

In summary, this and previous studies provide insight into angiopoietin expression following injury. In the early phase postinjury BBB breakdown is associated with decreased expression of Ang1 which is constitutively expressed in endothelium and rapid upregulation of Ang2. The latter may be a factor in early BBB breakdown occurring within hours of the injury. By day 1, Ang2 colocalizes with endothelial active Caspase-3 suggesting that Ang2 has a role in endothelial apoptosis. The latter may be another factor in BBB breakdown. Later upregulation of VEGF-A with Ang2 leads to a florid angiogenic response. By day 6, expression of these growth factors are restored to resting levels and there is normal constitutive expression of Ang1 in cerebral endothelium. Understanding the molecular events following brain injury is critical in designing strategies to modulate repair following brain injury.

Figure 5 Confocal images obtained in single channels are shown in (a), (c) and (e) while merged images are shown in (b), (d) and (f). (a) Endothelial Ang2 (green) is present in a pial arteriole showing BBB breakdown to fibronectin (red, arrowhead) which is shown in the merged confocal image (b). (c) Pial vessel from a control rat showing lack of immunoreactivity for active Caspase-3. (d) Dual staining of a day 1 lesion shows mural active Caspase-3 immunoreactivity in a pial vessel. The endothelium is focally positive for Ang1 (green, arrowhead). A polymorphonuclear leukocyte in the vessel lumen shows cytoplasmic Ang1 (green) while those in the neuropil show colocalization of Ang1 and active Caspase-3 (yellow, large arrowhead). (e) Pial vessel at day 1 shows Ang2 (green) in the endothelium and in the cytoplasm of polymorphonuclear leukocytes. (f) The merged confocal image shows colocalization (yellow) of Ang2 and active Caspase-3 in the endothelium and in the polymorphonuclear leukocytes (arrowheads) present in and around the vessel wall. Scale bar $=50 \mu \mathrm{m}$. 


\section{Acknowledgements}

This study is supported by the Heart and Stroke Foundation of Ontario. We thank Dr James H Eubanks for his invaluable appraisal and discussion of this work.

\section{References}

1 Davis S, Aldrich TH, Jones PF, et al. Isolation of angiopoietin-1, a ligand for the TIE2 receptor, by secretion-trap expression cloning. Cell 1996;87: 1161-1169.

2 Maisonpierre PC, Suri C, Jones PF, et al. Angiopoietin2 , a natural antagonist for Tie2 that disrupts in vivo angiogenesis. Science 1997;277:55-60.

3 Valenzuela DM, Griffiths JA, Rojas J, et al. Angiopoietins 3 and 4: diverging gene counterparts in mice and humans. Proc Natl Acad Sci USA 1999;96:1904-1909.

4 Jones PF. Not just angiogenesis-wider roles for the angiopoietins. J Pathol 2003;201:515-527.

5 Koh GY, Kim I, Kwak HJ, et al. Biomedical significance of endothelial cell specific growth factor, angiopoietin. Exp Mol Med 2002;34:1-11.

6 Hayes AJ, Huang WQ, Mallah J, et al. Angiopoietin-1 and its receptor Tie-2 participate in the regulation of capillary-like tubule formation and survival of endothelial cells. Microvascular Res 1999;58:224-237.

7 Kwak HJ, So JN, Lee SJ, et al. Angiopoietin-1 is an apoptosis survival factor for endothelial cells. Febs Lett 1999;448:249-253.

8 Papapetropoulos A, Fulton D, Mahboubi K, et al. Angiopoietin-1 inhibits endothelial cell apoptosis via the Akt/survivin pathway. J Biol Chem 2000;275: 9102-9105.

9 Cohen B, Barkan D, Levy Y, et al. Leptin induces angiopoietin-2 expression in adipose tissues. J Biol Chem 2001;276:7697-7700.

10 Zagzag D, Amirnovin R, Greco MA, et al. Vascular apoptosis and involution in gliomas precede neovascularization: a novel concept for glioma growth and angiogenesis. Lab Invest 2000;80:837-849.

11 Patan S. TIE1 and TIE2 receptor tyrosine kinases inversely regulate embryonic angiogenesis by the mechanism of intussusceptive microvascular growth. Microvasc Res 1998;56:1-21.

12 Suri C, Jones PF, Patan S, et al. Requisite role of angiopoietin-1, a ligand for the TIE2 receptor, during embryonic angiogenesis. Cell 1996;87:1171-1180.

13 Holash J, Wiegand SJ, Yancopoulos GD. New model of tumor angiogenesis: dynamic balance between vessel regression and growth mediated by angiopoietins and VEGF. Oncogene 1999;18:5356-5362.

14 Lobov IB, Brooks PC, Lang RA. Angiopoietin-2 displays VEGF-dependent modulation of capillary structure and endothelial cell survival in vivo. Proc Natl Acad Sci USA 2002;99:11205-11210.

15 Gamble JR, Drew J, Trezise L, et al. Angiopoietin-1 is an antipermeability and anti-inflammatory agent in vitro and targets cell junctions. Circ Res 2000;87: 603-607.
16 Thurston G, Suri C, Smith K, et al. Leakage-resistant blood vessels in mice transgenically overexpressing angiopoietin-1. Science 1999;286:2511-2514.

17 Nourhaghighi N, Teichert-Kuliszewska K, Davis J, et al. Altered expression of angiopoietins during bloodbrain barrier breakdown and angiogenesis. Lab Invest 2003;83:1211-1222.

18 Nag S, Takahashi JL, Kilty DW. Role of vascular endothelial growth factor in blood-brain barrier breakdown and angiogenesis in brain trauma. J Neuropathol Exp Neurol 1997;56:912-921.

19 Dobrogowska DH, Lossinsky AS, Tarnawski M, et al. Increased blood-brain barrier permeability and endothelial abnormalities induced by vascular endothelial growth factor. J Neurocytol 1998;27:163-173.

20 Wang W, Dentler WL, Borchardt RT. VEGF increases BMEC monolayer permeability by affecting occludin expression and tight junction assembly. Am J Physiol Heart Circ Physiol 2001;280:H434-H440.

21 Nag S. Blood-brain barrier permeability using tracers and immunohistochemistry. Methods Mol Med 2003; 89:133-144.

22 Nag S, Picard P, Stewart DJ. Expression of nitric oxide synthases and nitrotyrosine during blood-brain barrier breakdown and repair after cold injury. Lab Invest 2001;81:41-49.

23 Nag S, Eskandarian MR, Davis J, et al. Differential expression of vascular endothelial growth factor-A (VEGF-A) and VEGF-B after brain injury. J Neuropathol Exp Neurol 2002;61:778-788.

24 Gavrieli Y, Sherman Y, Ben Sasson SA. Identification of programmed cell death in situ via specific labeling of nuclear DNA fragmentation. J Cell Biol 1992;119: 493-501.

25 Nag S. Cold-injury of the cerebral cortex: immunolocalization of cellular proteins and blood-brain barrier permeability studies. J Neuropathol Exp Neurol 1996;55:880-888.

26 Nag S. The blood-brain barrier and cerebral angiogenesis: lessons from the cold-injury model. Trends Mol Med 2002;8:38-44.

27 Reichel A, Begley DJ, Abbott NJ. An overview of in vitro techniques for blood-brain barrier studies. Methods Mol Med 2003;89:307-324.

28 Teichert-Kuliszewska K, Maisonpierre PC, Jones N, et al. Biological action of angiopoietin-2 in a fibrin matrix model of angiogenesis is associated with activation of Tie2. Cardiovasc Res 2001;49:659-670.

29 Charriaut-Marlangue C, Ben Ari Y. A cautionary note on the use of the TUNEL stain to determine apoptosis. Neuroreport 1995;7:61-64.

30 Murakami K, Kondo T, Sato S, et al. Occurrence of apoptosis following cold injury-induced brain edema in mice. Neuroscience 1997;81:231-237.

31 Tominaga T, Kure S, Yoshimoto T. DNA fragmentation in focal cortical freeze injury of rats. Neurosci Lett 1992;139:265-268.

32 Beck H, Acker T, Wiessner C, et al. Expression of angiopoietin-1, angiopoietin-2, and tie receptors after middle cerebral artery occlusion in the rat. Amer J Pathol 2000;157:1473-1483.

33 Klagsbrun M, Moses MA. Molecular angiogenesis. Chem Biol 1999;6:R217-R224. 